\title{
Osmotic Water Permeation through a Carbon \\ Nanotube
}

Chang Fang, Decai Huang, Jiaye $\mathrm{Su}^{*}$

Department of Applied Physics, Nanjing University of Science and Technology, Nanjing, Jiangsu 210094, China.

*Electronic mail: jysu@iccas.ac.cn; jysu@ njust.edu.cn;

\section{$\underline{\text { Supporting information }}$}

\section{Model and simulation method}

A snapshot of the simulation model is shown in Figure 1 of the main text. As we have analyzed, the major difficult of building an osmotic model for long-time simulations is to separate the pure water and ionic solution in a periodic box. To address this question, we introduce a dipalmitoylphosphatidylcholine (DPPC) bilayer (3600 atoms) with the same size as graphene sheet $\left(5.0 \times 5.0 \mathrm{~nm}^{2}\right)$ to divide the pure water and salt solution compartments. As seen in Figure 1, the salt solution compartment exists in-between the right graphene and DPPC bilayer, which contains roughly 2700 water molecules with varying $\mathrm{NaCl}$ number; while the pure water (4500 water molecules) is in-between the DPPC bilayer and left graphene, through the PBC connection. A single CNT (2.56 nm in length with different diameters) connects the pure water and salt solution. As the DPPC bilayer is almost impermeable [S1], the CNT becomes a solely osmotic transporter for water. Due to the osmotic force, the pure water will be driven through the CNT to the salt solution compartment, and the position of DPPC bilayer will be adjusted automatically due to the compartment size. The whole simulation box contains roughly 28000 atoms with a size of $5.0 \times 5.0 \times 15.0$ $\mathrm{nm}^{3}$. Following the interest of recent experiments [S2,S3], we focus on the effect of salt concentration gradient (concentration gradient), temperature and CNT diameter. 
As the single CNT transports water at relatively low rates, our setup provides a simple, robust and long-time osmotic simulation model for fundamental study.

We conducted all the MD simulations by using Gromacs 4.6.7 simulation package [S4] at constant pressure (1 bar) and temperature (300 $\mathrm{K}$ if not specified). The water molecules were described by the classical SPC/E model [S5]. The CNT and graphene were modeled by uncharged Lennard-Jones (LJ) particles in the Amber 03 force field [S6]. LJ parameters of $\mathrm{NaCl}$ were taken from a previous work [S7]. The DPPC molecules were described by the Gromos 54a7 force field [S8,S9]. All the LJ interactions were cut-off at $1.2 \mathrm{~nm}$ and the particle-mesh Ewald (PME) method [S10] was employed to treat the long-range electrostatic interactions also with a $1.2 \mathrm{~nm}$ cut-off in real space. Periodic boundary condition (PBC) was applied in three dimensions. The MD time-step was set to 2.5 fs and data were collected every 1 ps. We performed two independent $125 \mathrm{~ns}$ simulations and the last $120 \mathrm{~ns}$ were used for data analysis. Standard error bars shown in the following plots were estimated by the two data points.

\section{The translocated water number with simulation time}

As seen in Figure S1, the translocated water number into the salt compartment increase almost linearly with the simulation time. As presented in our manuscript, the osmotic flux is defined as the net water number per nanosecond conducting across the CNT from the pure water to salt solution. Thus, the water flux is the ratio between the final translocated water number and time duration (120 ns). It deserves to note that the concentration gradient is corresponding to the initial values before the MD runs, i.e., it is calculated by the initial number of water and ions in the salt solution compartment. The number of water molecules in the salt solution compartment will increase with the simulation time, which leads to the decrease of concentration gradient. For the sake of simplicity, we use these initial values in the HP equation to estimate the osmotic flux. Therefore, compared to the reduced concentration gradient in simulations, the theoretical flux should be slightly overestimated. However, as the 
present comparison between simulation and HP equation should be qualitative, the theoretical estimation should not affect any conclusion.

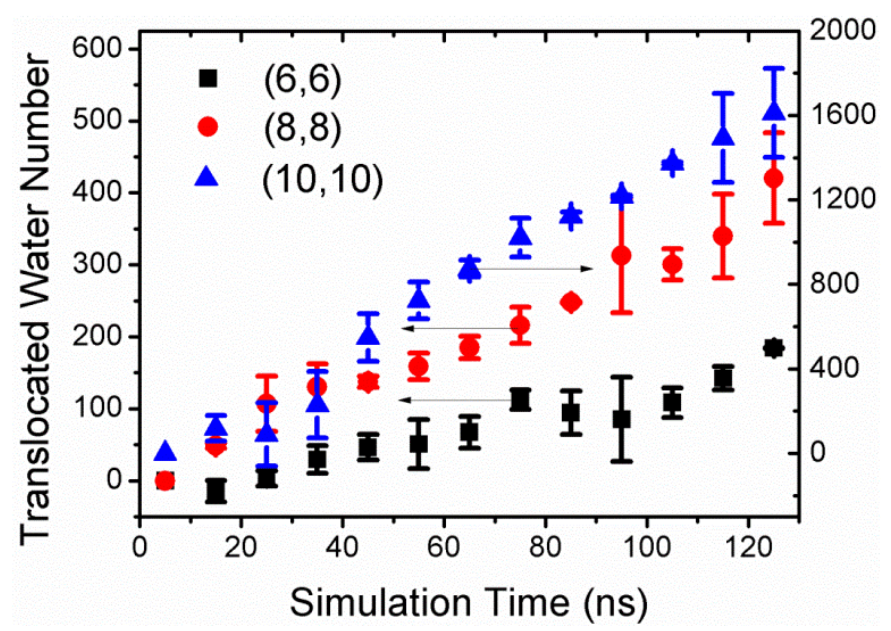

Figure S1. The water number translocated into the slat compartment as a function of the simulation time, where $\Delta C_{S}=2.03 \mathrm{M}$ and $\mathrm{T}=300 \mathrm{~K}$.

\section{The water translocation time with concentration gradient}

Figure $\mathrm{S} 2$ shows the water translocation time $\tau$ that is defined as the average time of individual water molecules crossing though the CNT successfully. Normally, the translocation time should be inversely proportional to the water flux, because the higher flux requires the quicker crossing process of water through the CNT. However, the translocation time herein is insensitive to the ionic concentration. We believe this should be caused by the thermal fluctuations as there exists considerable water flux against the osmotic pressure. Therefore, the osmotic pressure should be not a driving force that is strong enough to significantly reduce the thermal fluctuations. Generally, the single-file water chain inside CNT $(6,6)$ has to move collectively and thus should correspond to slow conduction; while the water molecules in wider CNTs can pass over with each other. Nonetheless, the CNT $(8,8)$ has an abnormal large translocation time even than the CNT $(6,6)$. The major reason is that the water inside CNT $(8,8)$ forms ice-like structures [S11] with low diffusion coefficient [S12], and will be discussed in the main text further. 


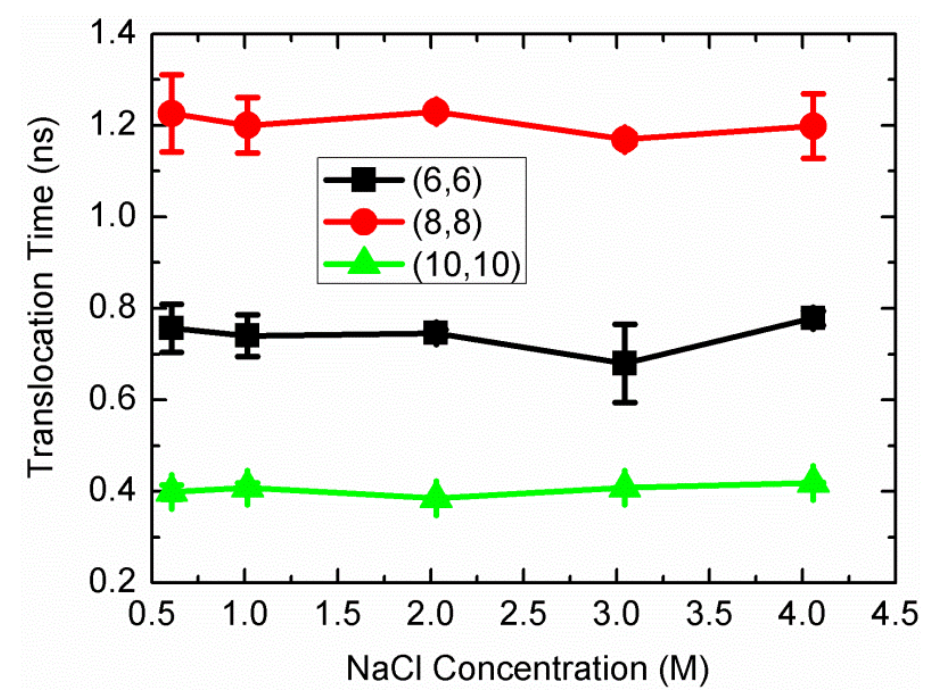

Figure S2. The water translocation time $\tau$ as a function of $\mathrm{NaCl}$ concentration with different type of CNTs at $\mathrm{T}=300 \mathrm{~K} . \tau$ indicates the average traveling time of individual water across the whole CNT.

\section{The water occupancy, density profiles and hydrogen bond for different concentration gradients}

The number of water molecules inside CNTs is usually at a constant value because water fills CNTs spontaneously and rearrange to special structures. Figure S3a shows the water occupancy $\langle\mathrm{N}\rangle$ inside the $\mathrm{CNT}$ as a function of the $\mathrm{NaCl}$ concentration. The fitting slopes manifests that the water occupancy depends on the salt concentration very weakly, but clearly depends on the CNT types. The diameters of $(6,6),(8,8)$ and $(10,10)$ CNTs are $0.81 \mathrm{~nm}, 1.08 \mathrm{~nm}$ and $1.35 \mathrm{~nm}$, respectively. The occupancy number inside these CNTs are nearly are 10, 35 and 62, accordingly. The confined water forms unique structures in different CNTs: single-file chain and vapor-like phase stabilized by entropy inside $(6,6) \mathrm{CNT}$, ice-like phase stabilized by enthalpy inside $(8,8) \mathrm{CNT}$, and bulk-like phase stabilized by large translational entropy [S13]. Water molecules inside $(6,6) \mathrm{CNT}$ centrally distribute in the center of the tube [S14]. The corresponding density shows wave-like pattern with ten ultra-high peaks in Figure S3b that are independent of the ionic concentration, similar to the 
water structure in aquaporins [S15]. A H-bond between two water molecules can be defined when the two oxygen distance is less than $0.35 \mathrm{~nm}$ and the angle between $\mathrm{O}-\mathrm{O}$ bond and any one of the $\mathrm{O}-\mathrm{H}$ bond is less than $30^{\circ}$, also exhibited in Figure $\mathrm{S} 3 \mathrm{~b}$. Remarkably, only at the solution side the number of H-bonds decreases with the increase of ionic concentration. This is because ions in solutions form hydrated ion clusters, which interrupt hydrogen bonds.

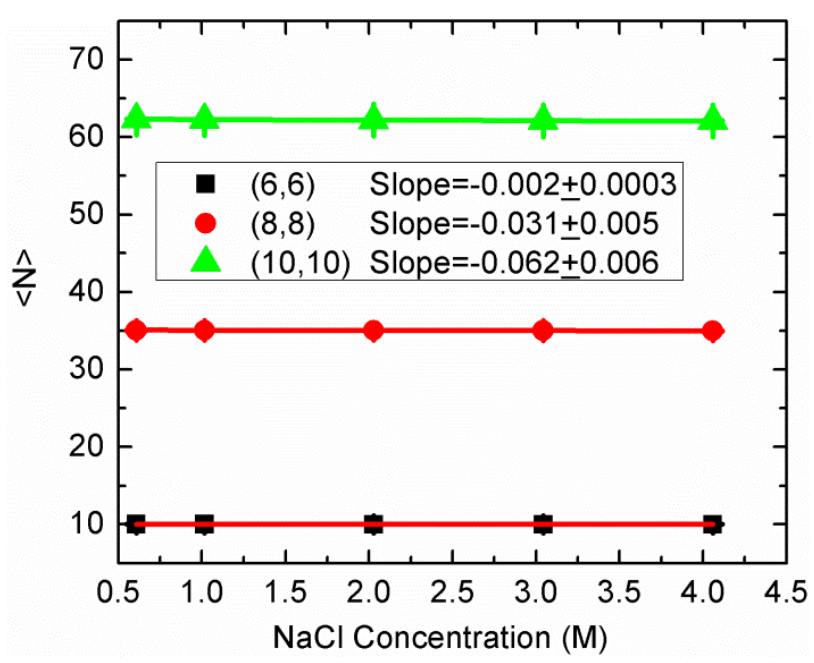

(a)

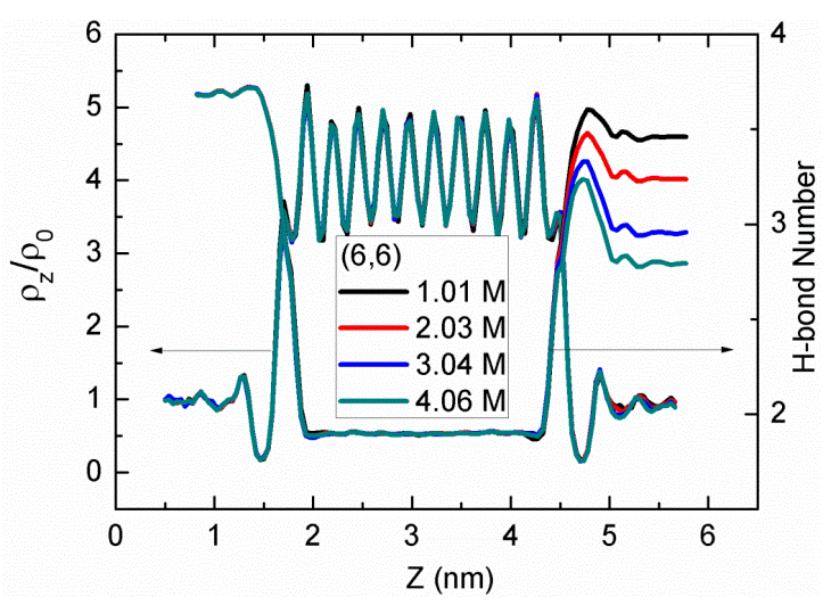

(b)

Figure S3. (a) The average water occupancy inside different CNTs as a function of $\mathrm{NaCl}$ concentration, and (b) the axial water density and hydrogen bond (H-bond) number as a function of position for the $\operatorname{CNT}(6,6)$ at $\mathrm{T}=300 \mathrm{~K} . \rho_{0}=1.0 \mathrm{~g} / \mathrm{cm}^{3}$ is the bulk water density. 


\section{The water occupancy, density profiles and hydrogen bond for different temperatures}

With the increase of temperature, thermal motion of water molecules becomes more intensive, leading to the decrease of the water occupancy inside CNTs, shown in Figure S4a. In the $(6,6) \mathrm{CNT}$, the occupancy $\langle\mathrm{N}\rangle$ is nearly fixed at the value of 10 due to the preservation of single-file water chain. Slight reduction occurs for the water occupancy in wider tubes. This reduction should be a result of the volume expansion caused by temperature increase, similar to the dehydration phenomenon in our daily life. The temperature also affects the water density and H-bond number, shown in Figure $\mathrm{S} 4 \mathrm{~b}$ for the $(6,6) \mathrm{CNT}$. With the increase of temperature, the wave-like density pattern becomes less intensive and the H-bond number also slightly decreases. This is clearly caused by the intensive motion of water; however, the change is still very limited as the single-file structure is not broken.

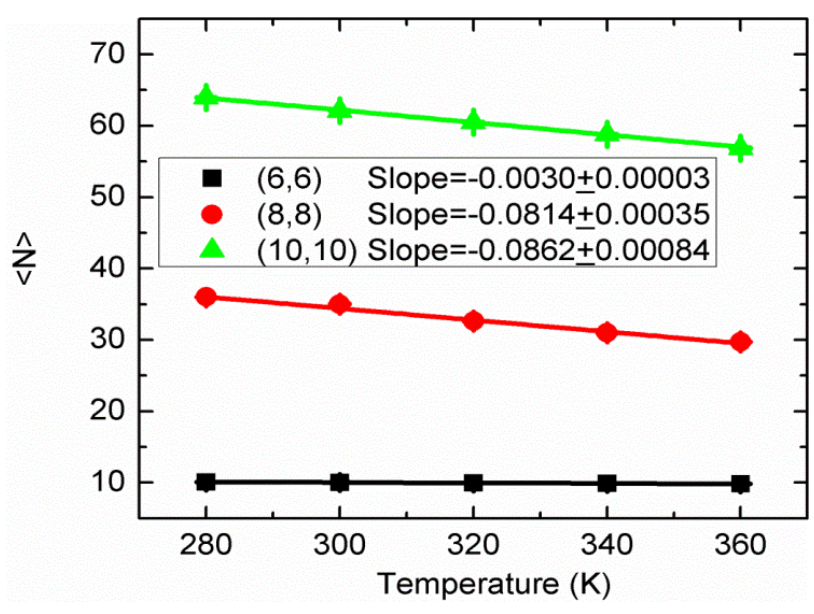

(a) 


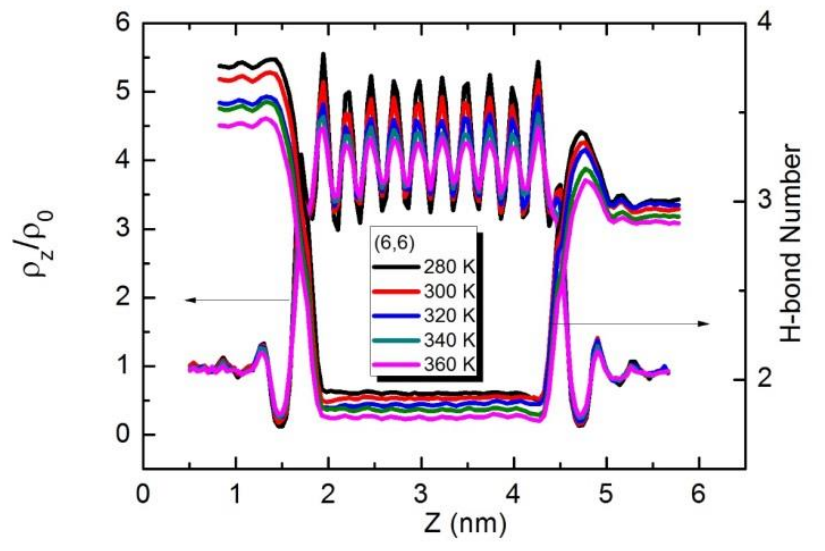

(b)

Figure S4. (a) The average water occupancy $\langle N\rangle$ as a function of temperature for different CNTs. (b) The Axial water density profiles and H-bond number for the CNT $(6,6)$. Here, $\Delta C_{S}=3.04 \mathrm{M}$.

\section{The water dipole distribution for different CNTs}

The water dipole orientation in Figure S5 demonstrates the great impact of CNT diameter. For the CNT $(6,6)$, the single-file water chain exhibits a standard two-peak distribution that is similar to the previous work [S14]. For the CNT $(8,8)$, the distribution becomes a sharp peak with two small shoulder peaks, due to the ice-like water structures discussed above. The distribution becomes a single peak and more sharp for further increasing the CNT diameter. It deserves to note that for the CNT $(10,10)$ and above, the dipole orientation will become more random and bulk-like, corresponding to a $90^{\circ}$ peak with respect to any axis. However, as we have shown in Figure 3c, the dipole of CNT $(8,8)$ is perpendicular to the CNT axis, which is a "real" $90^{\circ}$ peak. This should be why the peak of CNT $(8,8)$ is more narrow and sharp. 


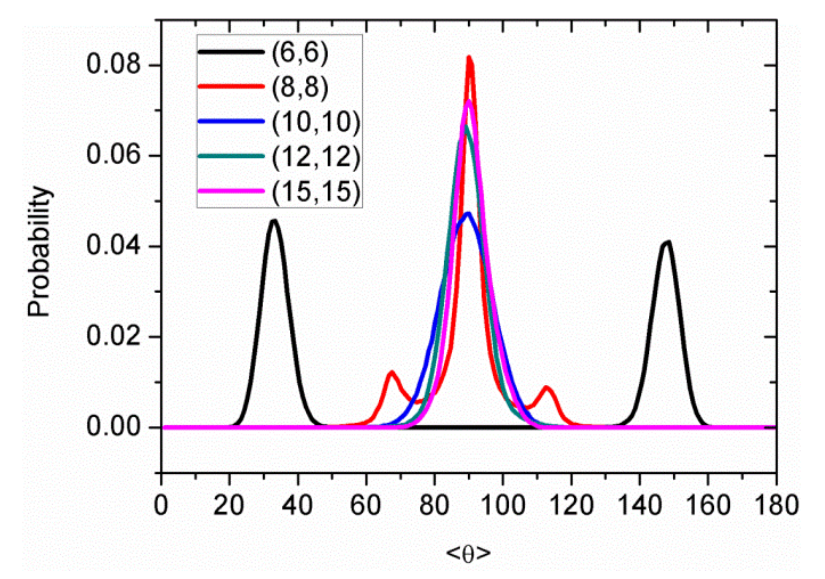

Figure S5. Probability distributions of water dipole orientation inside different CNTs, where $\Delta C_{S}=3.04 \mathrm{M}$ and $\mathrm{T}=300 \mathrm{~K}$.

\section{References}

[S1] Qiao, B.; Olvera de la Cruz, M. Driving Force for Water Permeation Across Lipid Membranes. J. Phys. Chem. Lett. 2013, 4, 3233-3237.

[S2] Tunuguntla, R. H.; Henley, R. Y.; Yao, Y. C.; Pham, T. A.; Wanunu, M.; Noy, A. Enhanced Water Permeability and Tunable Ion Selectivity in Subnanometer Carbon Nanotube Porins. Science 2017, 357, 792-796.

[S3] Lokesh, M.; Youn, S. K.; Park, H. G. Osmotic Transport across Surface Functionalized Carbon Nanotube Membrane. Nano Lett. 2018, 18, 6679-6685.

[S4] Hess, B.; Kutzner, C.; van der Spoel, D.; Lindahl, E. GROMACS 4: Algorithms for Highly Efficient, Load-Balanced, and Scalable Molecular Simulation. J. Chem. Theory Comput. 2008, 4, 435-447.

[S5] Berendsen, H. J. C.; Grigera, J. R.; Straatsma, T. P. The Missing Term in Effective Pair Potentials. J. Phys. Chem. 1987, 91, 6269-6271.

[S6] Duan, Y.; Wu, C.; Chowdhury, S.; Lee, M. C.; Xiong, G. M.; Zhang, W.; Yang, R.; Cieplak, P.; Luo, R.; Lee, T.; Caldwell, J.; Wang, J. M.; Kollman, P. A Point-Charge Force Field for Molecular Mechanics Simulations of Proteins Based on 
Condensed-Phase Quantum Mechanical Calculations. J. Comput. Chem. 2003, 24, 1999-2012.

[S7] Chowdhuri, S.; Chandra, A. Hydration Structure and Diffusion of Ions in Supercooled Water: Ion Size Effects. J. Chem. Phys. 2003, 118, 9719-9725.

[S8] Poger, D.; van Gunsteren, W. F.; Mark, A. E. A New Force Field for Simulating Phosphatidylcholine Bilayers. J. Comput. Chem. 2010, 31, 1117-1125.

[S9] Poger, D.; Mark, A. E. On the Validation of Molecular Dynamics Simulations of

Saturated and cis-Monounsaturated Phosphatidylcholine Lipid Bilayers: A Comparison with Experiment. J. Chem. Theory Comput. 2010, 6, 325-336.

[S10] Essmann, U.; Perera, L.; Berkowitz, M. L, Darden, T.; Lee, H.; Pedersen, L. G. A Smooth Particle Mesh Ewald Method. J. Chem. Phys. 1995, 103, 8577-8593.

[S11] He, Z.; Zhou, J. Lu, X.; Corry B. Ice-like Water Structure in Carbon Nanotube $(8,8)$ Induces Cationic Hydration Enhancement. J. Phys. Chem. C 2013, 117, $11412-11420$.

[S12] Farimani, A. B.; Aluru, N. R. Spatial Diffusion of Water in Carbon Nanotubes: From Fickian to Ballistic Motion. J. Phys. Chem. B 2011, 115, 12145-12149.

[S13] Pascal, T. A.; Goddard, W. A.; Jung, Y. Entropy and the Driving Force for the Filling of Carbon Nanotubes with Water. Proc. Natl. Acad. Sci. U. S. A. 2011, 108, 11794-11798.

[S14] Su, J.; Guo, H. Control of Unidirectional Transport of Single-File Water Molecules through Carbon Nanotubes in an Electric Field. ACS Nano 2011, 5, $351-359$.

[S15] de Groot, B. L.; Grubmüller, H. Water Permeation Across Biological Membranes: Mechanism and Dynamics of Aquaporin-1 and GlpF. Science 2001, 294, $2353-2357$. 